\title{
A Low-Cost Attack on Branch-Based Software Watermarking Schemes
}

\author{
Gaurav Gupta and Josef Pieprzyk \\ Centre for Advanced Computing - Algorithms and Cryptography, \\ Department of Computing, Division of Information and Communication Sciences, \\ Macquarie University, Sydney, NSW - 2109, \\ Australia \\ \{ggupta, josef\}@ics.mq.edu.au \\ http://www. comp.mq.edu.au/ ggupta
}

\begin{abstract}
In 2005, Ginger Myles and Hongxia Jin proposed a software watermarking scheme based on converting jump instructions or unconditional branch statements (UBSs) by calls to a fingerprint branch function (FBF) that computes the correct target address of the UBS as a function of the generated fingerprint and integrity check. If the program is tampered with, the fingerprint and integrity checks change and the target address will not be computed correctly. In this paper, we present an attack based on tracking stack pointer modifications to break the scheme and provide implementation details. The key element of the attack is to remove the fingerprint and integrity check generating code from the program after disassociating the target address from the fingerprint and integrity value. Using the debugging tools that give vast control to the attacker to track stack pointer operations, we perform both subtractive and watermark replacement attacks. The major steps in the attack are automated resulting in a fast and low-cost attack.
\end{abstract}

Keywords: software, watermark, unconditional branch, breakpoint.

\section{Introduction}

In recent years, watermarking and fingerprinting have gathered significant attention due to the growing concerns over digital piracy and forgery of multimedia documents including software. Fingerprinting and Software Authentication are two major security aspects that have emerged. While the former is related to preventing illegal distribution, the latter tries to ensure that the software has not been tampered with. Numerous models have been proposed with these objectives, embedding watermarks, fingerprints, and integrity checks in the source codes and/or executable codes. Software watermarking schemes can be classified as follows:

- Graph-based/branch-based software watermarking: The software is treated as a graph $G_{s}$ with sequential blocks of code as nodes and transfer instructions such as function calls and branch statements as edges connecting the nodes. 
The watermark is a separate code and realized as a graph $G_{w}$. The two graphs $G_{s}$ and $G_{w}$ are connected by inserting additional edges (implemented as branch statements). The resulting watermarked graph is $G_{s^{\prime}}=G_{s}+G_{w}$ and source code $s^{\prime}$ is decoded from $G_{s^{\prime}}$.

Venkatesan et al. 14 proposed the first graph-based software watermarking scheme. The central idea is to convert the software and the watermark code into digraphs and add new edges between the two graphs implemented by adding function calls between the software and watermark code. This scheme lacks error-correcting capabilities and is susceptible to re-ordering of instructions and addition of new function calls. Another problem in the scheme is that the random walk mentioned in the paper (refers to the next node to be added in the watermarked software graph being selected randomly from the software graph and the watermark graph) is not actually random. The node visited next is based on the number of remaining nodes belonging to software graph $N_{s}$ and the number of remaining nodes belonging to watermark graph $N_{w}$. The next node is chosen from the watermark nodes with a probability of $\frac{N_{w}}{N_{w}+N_{s}}$ and from the software nodes with a probability of $\frac{N_{s}}{N_{w}+N_{s}}$. In a typical scenario, $N_{s} \gg N_{w}$, hence the watermark is skewed towards the tail of the watermarked program. This information is useful for probabilistic attacks. Alternatively, a pseudo-random permutation of the nodes to be visited can be generated. For further literature in graphbased software watermarking, the reader is referred to 123413 . None of these schemes are completely secure against instruction and block re-ordering attacks.

- Register-based software watermarking: Registers used to store variables are changed depending on the watermark bit to be embedded by replacing higher level language code with an inline assembly code. The attacker intends to reallocate variables in registers if the watermark has to be removed. Though, no such attack has yet been proposed.

Register-based software watermarking based on the QP algorithm (named after authors $\mathrm{Qu}$ and Potkonjak) [1011] is presented in [7]. It modifies registers used to store variables depending on which variables are required at the same time. The scheme is susceptible to register re-allocation attacks. A secondary watermark destroys the old watermark and inserting bogus methods renders the original watermark useless by changing the interference graph.

- Thread-based software watermarking: Nagra et al. 9] propose encoding the watermark in the sequence of the threads that are executed. For example, there are 3 threads; $T_{1}, T_{2}, T_{3}, T_{1} \rightarrow T_{2} \rightarrow T_{3}$ encodes watermark $(000)_{2}$ and $T_{1} \rightarrow T_{3} \rightarrow T_{2}$ encodes watermark $(001)_{2}$ and so on. However, without any additional error-control mechanism, changing threads that execute piece of a code would destroy the watermark. Again, there has been no scheme claiming to break the watermark using suggested approach.

- Obfuscation-based software watermarking: This class of watermarking is applicable to object-oriented softwares. Class $C$ with functions $\left\{f_{1}, f_{2}, \ldots, f_{n}\right\}$ is partitioned into $k$ subclasses $\left\{C_{1}, C_{2}, \ldots, C_{k}\right\}$ and the watermark is 
encoded in the allocation of the functionalities Examples of such proposed schemes are 5612 .

This paper is organized as follows. Section 2 addresses related work in branchbased software watermarking and Section 3 describes watermarking scheme of Myles and Jin that we propose to attack. This is followed by a description of our attack in Section 4. Section 5 provides implementation details and results. We conclude our paper with a note on future enhancements in Section 6 .

\section{Related Work}

There have been several research projects dealing with branch-based software watermarking. These schemes exploit possibilities to modify the program's execution path by altering branch statements. In this section, we discuss two papers closely related to our attack. The first by Collberg et al. [1] that introduces Branch Functions. The second paper is by Myles and Jin [8] and describes the watermarking scheme that we attack in this paper.

Collberg et al. introduce the notion of Branch Function [1. Jump instructions or unconditional branch statements (UBSs) are replaced by calls to the branch function (for the sake of consistency, by branch, we mean an unconditional branch statement from now on) and modifies its own return address in order to return the control to the target of the branch statement. Figure 1 illustrates this process. If the program contains a jump instruction from $l_{\text {begin }}$ to $l_{\text {end }}$, several intermediate pit stops are inserted so that the control-flow graph becomes $l_{\text {begin }} \rightarrow a_{1} \rightarrow a_{2} \rightarrow \ldots \rightarrow l_{\text {end }}$ such that $l_{\text {begin }}$ has a jump instruction to $a_{1}$ which has a jump instruction to $a_{2}$ and so on. The pit stops are inserted using the rule:

$\operatorname{address}\left(a_{i}\right)<\operatorname{address}\left(a_{i+1}\right)$, if watermark bit $w_{i}=1$

$\operatorname{address}\left(a_{i}\right)>\operatorname{address}\left(a_{i+1}\right)$, if watermark bit $w_{i}=0$

Finally all the jump instructions are replaced by call to the branch function that determines the correct target address based on the calling address and returns the control to it.

Obvious attacks on such a scheme are adding an additional pit stop to the chain $l_{\text {begin }} \rightarrow a_{1} \rightarrow a_{2} \rightarrow \ldots \rightarrow l_{\text {end }}$ such that it becomes $l_{\text {begin }} \rightarrow a_{1} \rightarrow$ $a_{\text {extra }} \rightarrow a_{2} \rightarrow \ldots \rightarrow l_{\text {end }}$ or deleting an existing pit stop such that it becomes $l_{\text {begin }} \rightarrow a_{2} \rightarrow \ldots \rightarrow l_{\text {end }}$. The goal is to disturb the chain (thereby modify the watermark) yet keep the origin and target the same (hence keeping the execution path intact). Making similar changes, inserting secondary watermark is a trivial.

Myles and Jin propose an alternative fingerprinting model in [8]. The underlying concept remains the same, that is, a branch function transferring control to the target of the UBS, but in this case, the branch function contains the fingerprint-generating code, hence the name Fingerprint Branch Function $(\mathrm{FBF})$. FBF also computes an integrity check on the source code to ensure that 

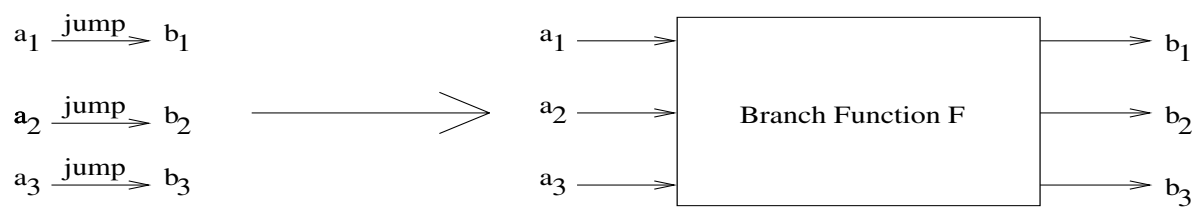

Fig. 1. Insertion of branch function $F$ that changes the return address according to the calling address and transfers control to target of the UBS. The jump instructions are now replaced by calls to $F$.

it is not modified. In the following section, we discuss this scheme in detail and analyze its flaws and weaknesses.

\section{Discussion on Watermarking Scheme}

The watermarking scheme is applied to software containing branch statements. These statements are then replaced by calls to FBF which returns control to the target address. The target address is generated from a recursive process of deriving new keys from old keys and checking the program for integrity. Additionally, an integrity check branch function (ICBF) is inserted in the program that verifies the integrity of FBF. If the user manipulates the program, the keys derived and integrity check value would change and hence the target address will change. The modified target address can be valid (belonging to code section of the program) which will result in incorrect execution of the program. Alternatively the target address can be invalid (lying outside the code section) resulting in runtime error. We will now discuss the two algorithms in the scheme, "embed" that inserts the watermark in the software and "recognize" that extracts the watermark from the watermarked software. They are defined as:

1. $\operatorname{embed}\left(P, A M\right.$, key $\left._{A M}, k e y_{F M}\right) \rightarrow P^{\prime}, F M$

2. $\operatorname{recognize}\left(P^{\prime}\right.$, key $_{A M}$, key $\left._{F M}\right) \rightarrow A M, F M$

where

$-P$ is the original software,

- $A M$ is the authorship mark,

- $k e y_{A M}$ is the secret input sequence to generate a trace of the program used to embed the watermark - the same for all copies of watermarked software,

- $k e y_{F M}$ is an initial secret key for deriving further keys - different for each copy of the watermarked program,

- $F M$ is the fingerprint mark

$-P^{\prime}$ is the watermarked software

\subsection{Watermark Embedding}

The steps involved in the embed algorithm are:

1. Let $\alpha$ be the set of all functions in $P$. Run the program with a secret input sequence $k e y_{A M}$. 
2. Obtain set $F$ of functions that lie in the execution path when the program is run with input sequence key $y_{M}$, let $\beta=\alpha-F$.

3. The number of UBSs in functions that belong to $F$ is $n$ and the number of UBSs in functions from $\beta$ is $m$.

4. Insert the two integer arrays; $T$ of size $n$ and $R$ of size $m$ in the data section of the program.

PROGRAM P

\begin{tabular}{|c|c|}
\hline $\mathrm{f}_{1}$ & When executed with \\
\hline $\mathrm{f}_{2}$ & secret input sequence Key $\mathrm{AM}$ \\
\hline $\mathrm{f}_{3}$ & $v$ \\
\hline
\end{tabular}

Set $\mathrm{F}$ of functions executed

Fig. 2. The set of functions $F$ is executed when the program $\mathrm{P}$ is run with the secret input key $y_{A M}$

5. Compute displacement $d_{i}$ between source $s_{i}$ and target $t_{i}$ of UBSs in functions that belong to $F$, so for instructions of the form $s_{i}$ : jmp $t_{i}$, the displacement $d_{i}=t_{i}-s_{i}$

6. In the program $P$, insert FBF $\xi$ that performs the following tasks:

(a) Initializes $k_{0}=\operatorname{key}_{F M}$.

(b) For $1 \leq i \leq n$,

i. Computes integrity check value $v_{i}$.

ii. Computes key $k_{i}$ from $\left(k_{i-1}, v_{i}, A M\right)$ by applying a one-way hash function $S H A_{1}$.

$$
k_{i}=S H A_{1}\left[\left(k_{i-1} \bigoplus A M\right) \| v_{i}\right]
$$

(c) Stores $d_{i}$ at $h\left(k_{i}\right)^{t h}$ location in array $T\left(T\left[h\left(k_{i}\right)\right]=d_{i}\right)$ where $h$ is a hash function, $h:\left\{k_{1}, k_{2}, \ldots, k_{n}\right\} \rightarrow\{1,2, \ldots, m\}(n \leq m)$.

7. Compute displacements $e_{i}$ between source $s_{i}$ and target $t_{i}$ of UBSs in functions that belong to $\beta$.

8. Insert ICBF $\phi$ in the program that:

(a) Computes integrity check value $v_{i}$. This value confirms the integrity of code section of the program containing $\xi$. 
(b) Stores displacement $e_{i}$ in array $R$ at index computed as a one-way hash function of $v_{i}\left(R\left[h\left(u_{i}\right)\right]=e_{i}\right)$. The hash function $h$ is the same that was used in Step 6.(c).

9. Replace all UBSs in $F$ by calls to $\xi$ and UBSs in $\beta$ by calls to $\phi$.

The fingerprint is generated as the embedding process executes. The final fingerprint is combination of all derived keys $-F M=k_{1}\left\|k_{2}\right\| \ldots k_{n}$. Users $u_{i}, u_{j}$ have distinct initializing keys $k e y_{F M_{i}}, k e y_{F M_{j}}$, hence final fingerprints $F M_{i}, F M_{j}$ are different.

\subsection{Watermark Recognition}

The recognize algorithm is run with the inputs $P^{\prime}$, key $_{A M}, k e y_{F M}$ and outputs the authorship mark $A M$ and fingerprint mark $F M$. When the program is run with the secret input $k e y_{A M}$, the function set $F$ is executed which generates the fingerprint mark $F M=k_{1}\left\|k_{2}\right\| \ldots k_{n}$ by initializing $k_{0}=k e y_{F M}$ and deriving successive keys using Equation (1). The authorship mark $A M$ can be extracted by isolating the one-way hash function $k_{i}=S H A_{1}\left[\left(k_{i-1} \bigoplus A M\right) \| v_{i}\right]$.

\section{Proposed Attack}

Objective of the attacker is to convert the fingerprinted program $P^{\prime}$ to the original program $P$. Since the displacements in $T$ are permuted, determining the correct target address of UBSs is computationally infeasible. Even if the size of $T$ is small, the program can have error-guards that intentionally corrupt the program after a specific number of run-time errors, making hit-and-trial attack impossible. The function $\phi$ checks the integrity of $\xi$, adding to the security of the scheme and thereby making the attack more difficult.

In $\xi$, the integrity check is done and a key is generated. The key is then mapped to the index in the displacement array where the correct displacement is stored. Security of the scheme depends on the correct execution path being a function of keys and integrity checks. If the key generated or the integrity value is incorrect, the displacement is wrong, and therefore the execution path is wrong. We concentrate our attack on this dependence. As soon as we can disassociate the correct execution path from the keys and integrity check, the code generating keys and integrity check can be deleted. The authors of [8] claim that the attacker needs to analyze the data section of the program to notice any changes and read the displacement array. This claim is fallacious as an attacker can track register values, including the stack pointer (SP) at:

1. Entry point of $\xi: \mathrm{SP}=s p_{i_{1}}$

2. Exit/ Return instruction of $\xi: \mathrm{SP}=s p_{i_{2}}$

The difference $s p_{i_{2}}-s p_{i_{1}}$ gives the displacement value $d_{i}$. Identification of the instructions participating in fingerprint generation is also achievable. According to [8], "In the second phase of the algorithm, the branches in each function $f$ 
that belongs to $F$ are replaced by calls to the FBF". We can create a mapping of functions being called by other functions and thereby create sets of functions which all point to one particular function. $\xi$ can be identified by the stack-pointer modifying statements and the set $F$ can be identified as the set of functions calling $\xi$. Therefore, $k e y_{A M}$ is no longer required to identify the set of functions participating in watermarking. Within the set $F$, each instruction calling $\xi$ and having memory address $s p_{1}$ can now be replaced by an unconditional branch to the instruction at $s p_{2}$. This can be achieved using inline assembly programming. For example, in $\mathrm{C}++$, a user can make use of _asm blocks. As a result, the displacement and hence the correct target address is no longer a function of the key and integrity check.

An example of such a block modifying the stack pointer is given below,

$\begin{array}{ll}\text { _asm }\{ & \\ \text { 1: } & \text { pop ECX; } \\ \text { 2: } & \text { add ECX, dis; } \\ \text { 3: } & \text { push } E C X ; \\ \text { \} } & \end{array}$

In the above code, statement 1 extracts the current value of Stack Pointer into register ECX. Statement 2 adds the intended displacement dis to the popped value and statement 3 pushes back the modified value onto the Stack. The Stack Pointer now contains a modified return address. If dis is positive, the new address $a_{t}$ is greater than the original return address $a_{r}\left(a_{t}>a_{r}\right)$ and the control is transferred "forward". If it is negative $\left(a_{t}<a_{r}\right)$, control is transferred "backward". Observe that $\phi$ calls can similarly be replaced by the original UBSs.

After changing calls to $\xi$ and $\phi$ by UBSs, the two functions $(\xi, \phi)$ can be deleted. When the recognize algorithm is run with input $k e y_{A M}, k e y_{F M}$, the inputs are unused dead variables, the algorithm doesn't output the fingerprint mark $F M$ and the recognition algorithm fails. The resulting software is equivalent to an un-watermarked software.

Summarizing our described process, the steps performed by the attacker are:

1. Identify $\xi$ : This task is accomplished by locating stack-pointer modifying statements. For example, in $\mathrm{C} / \mathrm{C}++$, searching for _asm blocks. If a program contains multiple _asm blocks, the ones with modification operation on ESP (Stack Pointer) requires to be targeted.

2. Identify $F$ : After identifying $\xi$, the fact that only the functions that belong to $F$ call $\xi$ can be utilized to identify $F$.

3. Displacement computation: Stack pointer values are recorded at the entry and exit points of $\xi\left(s p_{i_{1}}\right.$ and $s p_{i_{2}}$ respectively) and displacement $d_{i}$ is equal to $s p_{i_{2}}-s p_{i_{1}}$. Target instructions are determined from calling instruction and displacement. In our implementation, we use breakpoints to track the register values. 
4. Replacement of $\xi$ calls to UBSs: If the purpose of the attack is to remove the watermark, the function calls to $\xi$ are replaced by UBSs to obtain the original watermarked code.

5. Creating a modified watermarked program: The attacker can embed his/her own authorship mark $A M^{\prime}$ after removing the original authorship mark $A M$. For a successful attack, $\left(A M^{\prime}, F M^{\prime}\right)$ should be recognized on running recognize algorithm with parameters $P^{\prime}$, key $_{F M}$, key $A M$ where $F M^{\prime} \neq F M$.

(a) For all $f$ that belong to $F$, compute the displacement between the calling address and the target address and store in an array along with the calling address.

(b) Replace the UBSs by call to a new Fingerprint Branch Function, $\widetilde{\xi}$.

(c) $\widetilde{\xi}$ need not compute integrity check but simple calculates a new key based on the old key and attacker's authorship mark AM'.

$$
k_{i}=S H A 1\left[k_{i-1} \bigoplus A M^{\prime}\right] .
$$

Comparing (1) and (2), $k_{i}^{\prime} \neq k_{i}, 1 \leq i \leq n$.

(d) Map the keys to correct displacement using hash,

$$
\begin{aligned}
& h:\left\{k_{1}^{\prime}, k_{2}^{\prime}, \ldots, k_{n}^{\prime}\right\} \rightarrow\{1,2, \ldots, m\}(n \leq m) \\
& T\left[h\left(k_{i}^{\prime}\right)\right]=d_{i}
\end{aligned}
$$

The key sequence $F M^{\prime}$ generated is different from the original key sequence $F M$ as the individual keys are different. More formally,

$$
\begin{aligned}
& k_{1}^{\prime} \neq k_{1}, k_{2}^{\prime} \neq k_{2}, \ldots, k_{n}^{\prime} \neq k_{n} \\
& \Rightarrow\left\{k_{1}^{\prime}, k_{2}^{\prime}, \ldots, k_{n}^{\prime}\right\} \neq\left\{k_{1}, k_{2}, \ldots, k_{n}\right\} \\
& \Rightarrow\left\{k_{1}^{\prime}, k_{2}^{\prime}, \ldots, k_{n}^{\prime}\right\} \neq F M \\
& \Rightarrow F M^{\prime} \neq F M
\end{aligned}
$$

The recognition algorithm now outputs $F M^{\prime}, A M^{\prime}$ when executed with the inputs $P^{\prime}$, key $y_{A M}$, key $y_{F M}$.

In terms of efficiency, the overall complexity of attack depends on complexities of steps 3 and 4 as others are one-off steps. Steps 3 and 4 have linear complexity and hence the attack has $O(n)$ complexity. Steps 1 and 2 are automated and no human inspection is required to identify $\xi$ and $F$.

\section{Implementation Details and Results}

We have implemented the watermarking scheme in Visual $\mathrm{C}++$ and carried out the attack using the same. The features useful in doing so are the debug lookup windows - disassembly and register. The stack pointer value can then 
be tracked by using breakpoints under debugging mode and there is minimal manual intervention or inspection required. The following is disassembled code of the watermarked program used to compute displacement values.

Function $f_{i}$ that belongs to $F$ calling $\mathrm{FBF} \xi$ in statement 94:

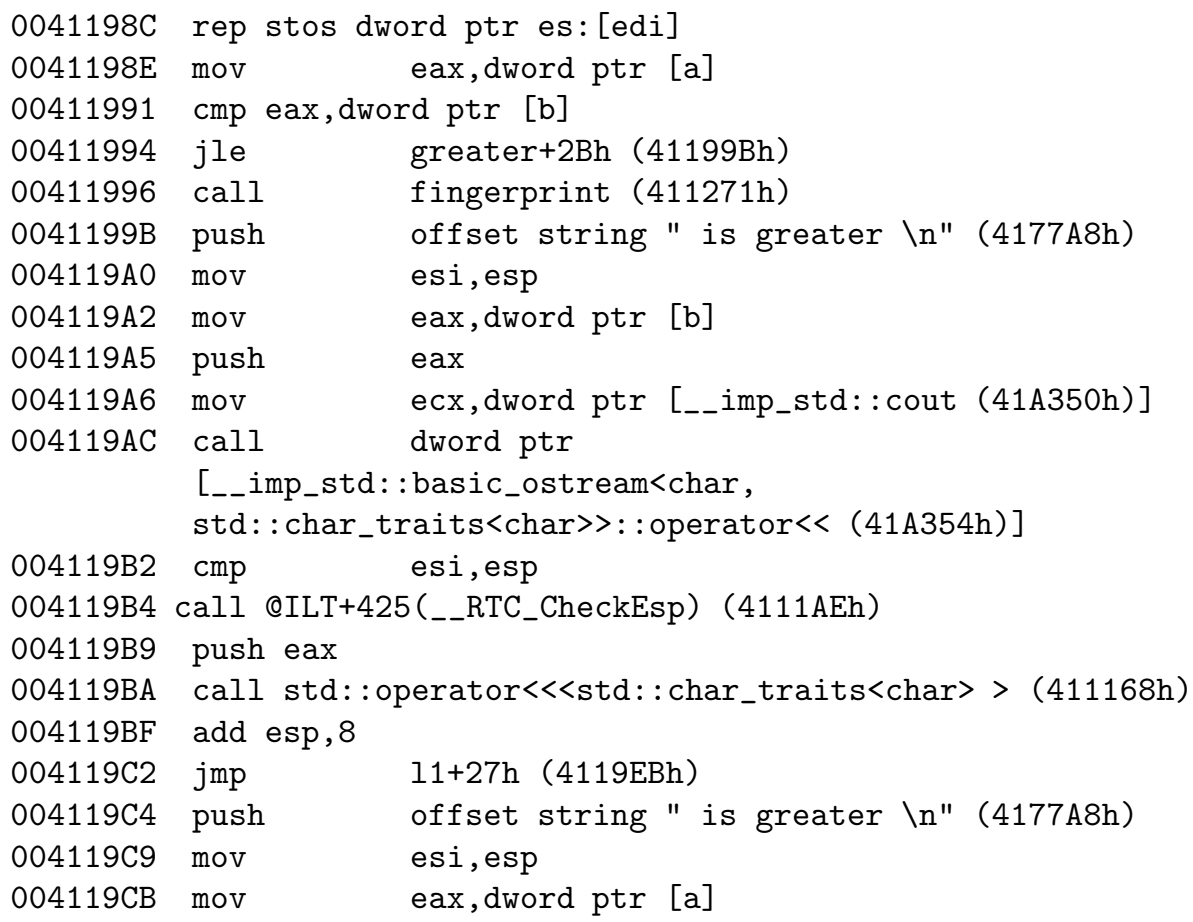

Fingerprint branch function code modifying return address:

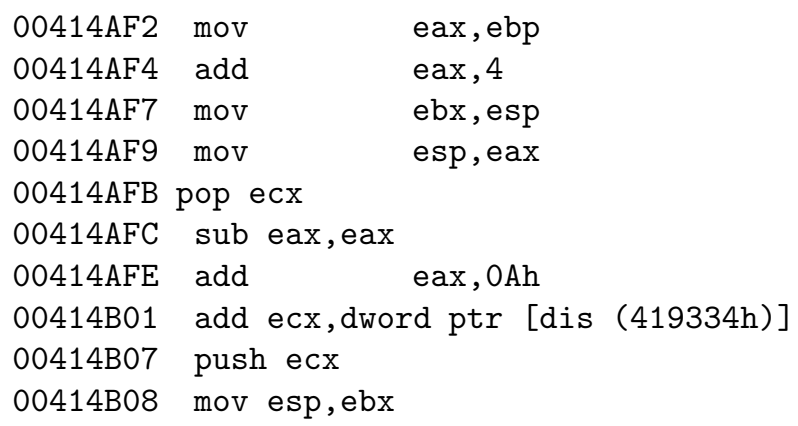

Register values are tracked while the program is executed and the following results are obtained:

Statement 00414AF2: EIP stores calling address, EIP $=00411996$. 
Statement 00414AFB: Return address, stored in the stack pointer, is popped into ECX, ECX = 0041199B.

Statement 00414B01: ECX adds displacement value to calling address, ECX = $004119 \mathrm{C} 4$.

Statement 00414B07: ECX value is pushed onto stack pointer. fingerprint(); returns control to this address.

In a nutshell, instruction 94 calls fingerprint(); which returns control to instruction 98 (the target of the original UBS) based on the value of dis looked up from array $T$. The attacker can thus compute the difference between $E C X$ value at statement $80\left(E C X_{80}\right)$ and $E C X$ value at statement $83\left(E C X_{83}\right)$ to find the value of displacement, then replace fingerprint(); call at statement 94 by UBS transferring control to $\Psi\left(\Phi(94)+E C X_{83}-E C X_{80}\right)$ (where $\Phi(x)$ denotes address of instruction $x$ and $\Psi(y)$ represents instruction at address $y$ ).

\section{Conclusion and Future Work}

In this paper, we present a successful low-cost attack on the branch-based watermarking scheme proposed in [8]. The cost of the attack is low in terms of hardware resources required since the only resources required are a functional computer with sufficient memory, storage and speed. The attack is efficient as manual inspection is required only during the step in which displacement values are noted from the disassembly register window. Even this is a debugger-specific constraint and in theory, it can be automated, however, we are unaware of an existing debugger that can perform this task. We provided an implementation of our scheme and some practical examples. The work lays a strong foundation for attacking similar software watermarking models [1/2/3.413 that depend on branching and inserting bogus functions in the program in order to embed a watermark. This paper also shows that tracking registers and branches is a trivial task using debugging tools and hence opens up a very interesting question of how can the watermarking schemes survive attacks with such advanced capabilities? Our future work is concerned with the following:

- We have shown that the attack is correct in theory and implemented a semi-automated version of the attack. We will work towards enhancing the implementation such that a fingerprinted program written in any language can be attacked. Practically, this is feasible since the attack operates on the disassembled code which, irrespective of the programming language in which it is written, is similar. However, the challenge would be to make the register tracking process compiler-independent. We also intend to design attacks for other branch-based watermarking schemes. Since the central security guard in such schemes is the dependency of the target address on integrity check and watermark values, they can be attacked in a manner similar to our attack. 
- Modifying scheme proposed in [8] so that the attack described in this paper is rendered ineffective by creating more complex dependency of inherent functionality of the program on the keys generated so that the attacker cannot remove fingerprint code without affecting the correct execution of the program. This can be done by introducing parameters other than displacement to bind the program's execution to the keys generated. As a simple example, a program may modify itself choosing from a set of modifications based on the key generated.

\section{Acknowledgements}

We would like to extend our appreciation for the contributions made by Saurabh Singh towards this research. The second author is supported by Australian Research Council grants DP0345366 and DF0451484.

\section{References}

1. Christian Collberg, Edward Carter, Saumya Debray, Andrew Huntwork, Cullen Linn, and Mike Stepp. Dynamic path-based software watermarking. In Proceedings of Conference on Programming Language Design and Implementation, volume 39, pages 107-118, June 2004.

2. Christian Collberg, Andrew Huntwork, Edward Carter, and Gregg Townsend. Graph theoretic software watermarks: Implementation, analysis, and attacks. In Proceedings of 6th Information Hiding Workshop, LNCS, volume 3200, pages 192 207, 2004.

3. Christian Collberg, Stephen Kobourov, Edward Carter, and Clark Thomborson. Error-correcting graphs for software watermarking. In Proceedings of 29th Workshop on Graph Theoretic Concepts in Computer Science, pages 156-167, 2003.

4. Christian Collberg and Clark Thomborson. Software watermarking: Models and dynamic embeddings. In Proceedings of Principles of Programming Languages 1999, POPL'99, pages 311-324, 1999.

5. Christian S. Collberg and Clark Thomborson. Watermarking, tamper-proofing, and obfuscation - tools for software protection. In IEEE Transactions on Software Engineering, volume 28, pages 735-746, August 2002.

6. Kazuhide Fukushima and Kouichi Sakurai. A software fingerprinting scheme for java using classfiles obfuscation. In Proceedings of Information Security Applications, LNCS, volume 2908, pages 303-316, 2004.

7. Ginger Myles and Christian Collberg. Software watermarking through register allocation: Implementation, analysis, and attacks. In Proceedings of International Conference on Information Security and Cryptology, LNCS, volume 2971, pages 274-293, 2003.

8. Ginger Myles and Hongxia Jin. Self-validating branch-based software watermarking. In Proceedings of 7th Information Hiding Workshop, LNCS, volume 3727, pages 342-356, 2005.

9. Jasvir Nagra and Clark Thomborson. Threading software watermarks. In Proceedings of 6th Information Hiding Workshop, LNCS, volume 3200, pages 208-223, 2004 . 
10. Gang Qu and Miodrag Potkonjak. Analysis of watermarking techniques for graph coloring problem. In Proceedings of International Conference on Computer Aided Design, pages 190-193, 1998.

11. Gang Qu and Miodrag Potkonjak. Hiding signatures in graph coloring solutions. In Proceedings of 3rd Information Hiding Workshop, LNCS, volume 1768, pages 348-367, 1999.

12. Mikhail Sosonkin, Gleb Naumovich, and Nasir Memon. Obfuscation of design intent in object-oriented applications. In Proceedings of 3rd ACM workshop on Digital Rights Management, pages 142-153, 2003.

13. Clark Thomborson, Jasvir Nagra, Ram Somaraju, and Charles He. Tamperproofing software watermarks. In Proceedings of Australasian Information Security Workshop, volume 32, pages 27-36, 2004.

14. Ramarathnam Venkatesan, Vijay Vazirani, and Saurabh Sinha. A graph theoretic approach to software watermarking. In Proceedings of 4 th Information Hiding Workshop, LNCS, volume 2137, pages 157-168, 2001. 